\title{
Metallicity evolution in mergers of disk galaxies with black holes
}

\author{
Antti Rantala and Peter H. Johansson \\ Department of Physics, University of Helsinki, \\ Gustaf Hällströmin katu 2a, 00560 Helsinki, Finland \\ antti.rantala@helsinki.fi \\ peter.johansson@helsinki.fi
}

\begin{abstract}
We use the TreeSPH simulation code Gadget-3 including a recently improved smoothed particle hydrodynamics (SPH) module, a detailed metallicity evolution model and sophisticated subresolution feedback models for supernovae and supermassive black holes in order to study the metallicity evolution in disk galaxy mergers. In addition, we examine the simulated morphology, star formation histories, metallicity gradients and kinematic properties of merging galaxies and merger remnants. We will compare our simulation results with observations of the early-type Centaurus A galaxy and the currently colliding Antennae galaxies.
\end{abstract}

Keywords. galaxies: abundances, galaxies: evolution, galaxies: formation, galaxies: interactions, methods: n-body simulations.

\section{Introduction}

A scenario has been put forward in which intermediate-mass, fast-rotating elliptical galaxies have been formed in mergers of disk galaxies (e.g. Naab et al. 2007, Johansson et al. 2009). The first galaxy interaction simulations by Toomre \& Toomre (1972) contained only collisionless N-body particles. The development of SPH (see Monaghan 1992 for a review and e.g. Hu et al. 2014 for recent improvements) and computationally inexpensive gravitational force algorithms (e.g. the tree code) led eventually to modern galaxy merger codes such as Gadget (Springel 2005). Present-day simulations containing subresolution astrophysical models for gas and stellar metallicity, star formation, supernova feedback (Scannapieco et al. 2006) and supermassive black hole (SMBH) feedback (Springel et al. 2005, Choi et al. 2014) enable detailed comparisons with observations.

\section{Metals in Merger Simulations}

Following the approach of Johansson et al. (2009), we construct two disk galaxies consisting of a dark matter halo, a rotationally supported disk of gas and stars, and a central bulge. Both the bulge and halo component have Hernquist-like density profiles while the disk component is of an exponential form. We employ both random orbital geometries for large simulation samples and specific orbital geometries set to match observed galaxy mergers, such as the Antennae galaxies (Karl et al. 2010). We set the initial fractional abundances of 10 metal species (C, N, O, Ne, Mg, Si, S, Ca and Fe) in the following way. Observations indicate that a radial metallicity gradient exists in disk galaxies. We use dex units $(\operatorname{dex}=12+\log 10(X / H))$ in which the radial abundance gradient is close to a linear form: $\operatorname{dex}(r)=\operatorname{dex}\left(r_{0}\right)+k\left(r-r_{0}\right)$, where $r_{0}$ is a scale radius and $\mathrm{k}$ is the negative metallicity gradient (Zaritsky et al. 1994, Kilian et al. 1994). The vertical abundance gradient is set in a similar way, motivated by observations from Ivezić et al. (2008). The ages of disk and bulge stars are initialized at $t=0$ from an exponentially 
decaying star formation rate $(\mathrm{SFR} \propto \exp (-t / \tau))$ with $\tau=1$ Gyr for bulge stars and $\tau=4$ Gyr for disk stars. The interstellar medium is enriched during the simulation by Type Ia and II supernovae and winds of AGB stars with metal yields calculated by Iwamoto et al. (1999), Woosley \& Weaver (1995) and Karakas (2010), respectively. Gas metallicity is smoothed using a metal diffusion technique between neighboring gas particles (Aumer et al. 2013).

\section{Ongoing and Future Work}

Currently (September 2014), the initial conditions of the merger simulations have been prepared and high-resolution simulations will be run in late 2014. We study especially the impact of SMBH feedback on the metallicity evolution in galaxy mergers, since previous studies have not considered both detailed metallicity evolution models and black holes at the same time. We also plan to study the triggering mechanisms of active galactic nuclei (AGN) in the aftermath of galaxy mergers as these systems with high $\mathrm{BH}$ accretion rates at high redshifts are possible quasar host galaxy candidates. We will produce simulated spectral energy distributions of quasar host galaxies using stellar population modelling techniques and compare the results with the observed properties of quasar host galaxies (Kotilainen et al. 2009).

\section{References}

Aumer, M., White, S. D. M., Naab, T., \& Scannapieco, C. 2013, MNRAS, 434, 3142

Choi, E., Naab, T., Ostriker, J. P., Johansson, P. H., \& Moster, B. P. 2014, MNRAS, 442, 440

Hu, C.-Y., Naab, T., Walch, S., Moster, B. P., \& Oser, L. 2014, MNRAS, 443, 1173

Johansson, P. H., Naab, T., \& Burkert, A. 2009a, ApJ, 690, 802

Ivezić, Ž., Sesar, B., Jurić, M., et al. 2008, ApJ, 684, 287

Iwamoto, K., Brachwitz, F., Nomoto, K., et al. 1999, ApJS, 125, 439

Karakas, A. I. 2010, MNRAS, 403, 1413

Karl, S. J., Naab, T., Johansson, P. H., et al. 2010, ApJ, 715, L88

Kilian, J., Montenbruck, O., \& Nissen, P. E. 1994, A\& $A, 284,437$

Kotilainen, J. K., Falomo, R., Decarli, R., et al. 2009, ApJ, 703, 1663

Monaghan, J. J. 1992, ARAESA, 30, 543

Naab, T., Johansson, P. H., Ostriker, J. P., \& Efstathiou, G. 2007, ApJ, 658, 710

Scannapieco, C., Tissera, P. B., White, S. D. M., \& Springel, V. 2006, MNRAS, 371, 1125

Springel, V. 2005, MNRAS, 364, 1105

Springel, V., Di Matteo, T., \& Hernquist, L. 2005, MNRAS, 361, 776

Toomre, A. \& Toomre, J. 1972, ApJ, 178, 623

Woosley, S. E. \& Weaver, T. A. 1995, ApJS, 101, 181

Zaritsky, D., Kennicutt, R. C., Jr., \& Huchra, J. P. 1994, ApJ, 420, 87 